\title{
What do we know about long laminar plasma jets?*
}

\author{
Xi Chen ${ }^{1, \ddagger}$, Wenxia Pan ${ }^{2}$, Xian Meng ${ }^{2}$, Kai Cheng ${ }^{1}$, Dong-Yan Xu , \\ and Chengkang $\mathrm{Wu}^{2}$
}

\author{
${ }^{1}$ Department of Engineering Mechanics, Tsinghua University, Beijing 100084, \\ China; ${ }^{2}$ Institute of Mechanics, Chinese Academy of Sciences, Beijing 100080 , \\ China
}

\begin{abstract}
Silent and stable long laminar plasma jets can be generated in a rather wide range of working parameters. The laminar flow state can be maintained even if considerable parameter fluctuations exist in the laminar plasma jet or if there is an impact of laterally injected particulate matter and its carrier gas. The attractive special features of laminar plasma jets include extremely low noise level, less entrainment of ambient air, much longer and adjustable high-temperature region length, and smaller axial gradient of plasma parameters. Modeling results show that the laminar plasma jet length increases with increasing jet inlet velocity or temperature and the effect of natural convection on laminar plasma jet characteristics can be ignored, consistent with experimental observations. The large difference between laminar and turbulent plasma jet characteristics is revealed to be due to their different laws of surrounding gas entrainment. Besides the promising applications of the laminar plasma jet to remelting and cladding strengthening of the metallic surface and to thermal barrier coating preparation, it is expected that the laminar plasma jet can become a rather ideal object for the basic studies of thermal plasma science owing to the nonexistence of the complexity caused by turbulence.
\end{abstract}

Keywords: thermal plasma jets; jet flow state; jet characteristics; carrier-gas impact; ambient air entrainment; buoyancy effects; applications; experiments; modeling.

\section{INTRODUCTION}

As is well known, cold laminar gas jets (e.g., at room temperature) can only maintain their laminar flow state within a short distance from the exit of the jet nozzle (usually, the jet laminar-region length/diameter ratio does not exceed 10) even at comparatively low Reynolds numbers [1,2]. Linear instability analysis [3] and experimental observation [2] showed that the cold laminar gas jet becomes even more unstable when the density of the gas jet is less than that of the ambient gas. Since the density of a plasma jet is always much less than that of the cold ambient air, one might anticipate that it is hard to generate a stable long laminar plasma jet in atmospheric-pressure air surroundings. Indeed, long laminar plasma jets cannot be generated by use of a conventional DC arc plasma torch employed for plasma spraying [4] or even for basic studies [5,6]. However, stable long laminar thermal plasma jets have been successfully generated in recent decades by the use of specially designed DC nontransferred arc plasma

\footnotetext{
*Paper presented at the $17^{\text {th }}$ International Symposium on Plasma Chemistry (ISPC 17), Toronto, Ontario, Canada, 7-12 August 2005. Other presentations are published in this issue, pp. 1093-1298.

¥Corresponding author: E-mail: cx-dem@mail.tsinghua.edu.cn
} 
torches in atmospheric-pressure air surroundings [7-9]. Experimental results $[9,10]$ showed that the long laminar plasma jets are characterized by stable flow state, negligible noise emission, and reduced entrainment of ambient air into the jet, thus leading to a rather long high-temperature region length (visual jet length/diameter ratio may be as great as 80 or even more), and the jet length can be easily adjusted by changing the flow rate of the working gas or the arc current. The long laminar plasma jet can be generated in a rather wide range of the working-gas flow rate and arc current of the arc plasma torch, and rather high jet inlet velocity $(\sim 1000 \mathrm{~m} / \mathrm{s})$ and temperature $(\sim 17000 \mathrm{~K})$ can be achieved. Preliminary attempts [10-14] in applying the laminar plasma jets to the preparation of thermal barrier coatings, to the remelting hardening of cast iron, and to the stainless-steel surface cladding showed encouraging results.

So far, our understanding of the laminar plasma jet characteristics is still incomplete. In comparison with the numerous studies of turbulent plasma jets (e.g., see [4,15] and refs. cited therein), not enough attention has been paid to the studies of the laminar plasma jet characteristics. In order to improve our understanding on long laminar plasma jet characteristics and stimulate its applications, this paper summarizes some recent research progress in this field.

\section{EXPERIMENTAL STUDIES}

A DC nontransferred arc plasma torch similar to that used in [9] has been employed in the present experimental study. Pure argon is used as the plasma-forming gas, and the generated plasma jet is issued into the atmospheric-pressure air surroundings. The working gas (argon) is admitted into the plasma torch axially and tangentially around the upstream end of the cathode and also tangentially into the torch from an annular slot near the downstream section of the inter-electrode insert of the arc plasma torch. In experiments, photographs of the plasma jets are taken from the lateral direction to determine the visual high-temperature region length of the plasma jet (which will be called jet length hereafter). Spectral-line intensity method is used to measure the plasma temperature distributions on the jet crosssection near the plasma torch exit. A small-size, taper-head, and water-cooled Pitot probe is used to measure the impact pressure and deduce the jet axial velocity. Rapid-response sensors are used to record and analyze the fluctuations of arc voltage, jet impact pressure, and spectral emission intensity.

Experimental results show that depending on working parameters of the plasma generation (arc current, working-gas flow rate, etc.), silent, long, stable, and laminar plasma jets or noisy, short, fluctuating, and turbulent plasma jets can be generated with the same DC arc plasma torch. Figure 1 summarizes the experimentally observed results concerning how the jet flow state and the jet length change with the argon flow rate and the arc current of the plasma torch. It is seen in Fig. 1 that for a given arc current, there exist two different critical values of the argon flow rate, i.e., $G_{1}$ and $G_{2}\left(G_{1}<G_{2}\right)$, demarcating the jet flow regimes. When the gas flow rate is less than the lower critical value $G_{1}$, the plasma jet assumes a silent and stable laminar flow state, and the jet length increases appreciably with increasing gas flow rate. When the working-gas flow rate is larger than the higher critical value $G_{2}$, the plasma jet assumes a noisy and fluctuating turbulent flow state, and the jet length is much shorter and almost does not depend on the working-gas flow rate. Between the laminar and turbulent flow regimes (i.e., with a flow rate less than $G_{2}$ but larger than $G_{1}$ ), the jet assumes an unsteady transitional state in which the plasma jet changes its flow state alternatively as a silent long jet or as a noisy short jet. The time-averaged jet length in the transitional state decreases with increasing gas flow rate, as shown in Fig. 1. Both the critical flow rates $G_{1}$ and $G_{2}$ (in $10^{-4} \mathrm{~kg} / \mathrm{s}$ ) increase linearly with increasing arc current $I$ (in A) according to the empirical correlations $G_{1}=0.024 I-1.10$ and $G_{2}=0.024 I+0.07$, which are plotted as two parallel straight lines, i.e., the full line for $G_{1}$ and the dashed line for $G_{2}$, respectively, in Fig. 1. The change of the jet flow state and the jet length with the argon flow rate for different arc currents can be more clearly seen in Fig. 2. Two straight lines demarcating the jet flow regimes are also plotted in Fig. 2, although the two lines are no longer parallel to each other in this figure. As is seen, in the laminar regime, the jet length increases notably with increasing arc current or increasing argon flow 


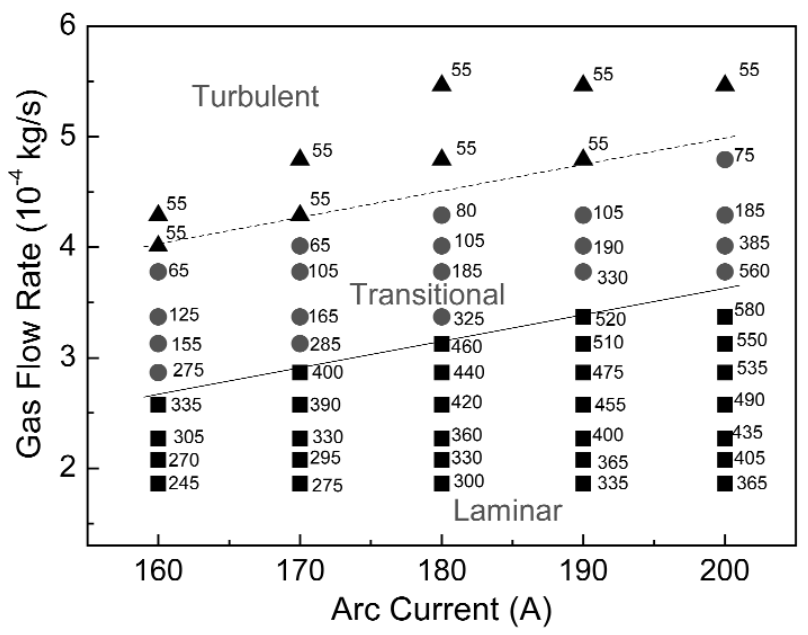

Fig. 1 Variations of the plasma jet flow state and the jet length with the argon flow rate and arc current of the DC arc plasma torch. Numerals denote jet lengths. Squares: laminar; circles: transitional; and triangles: turbulent regime.

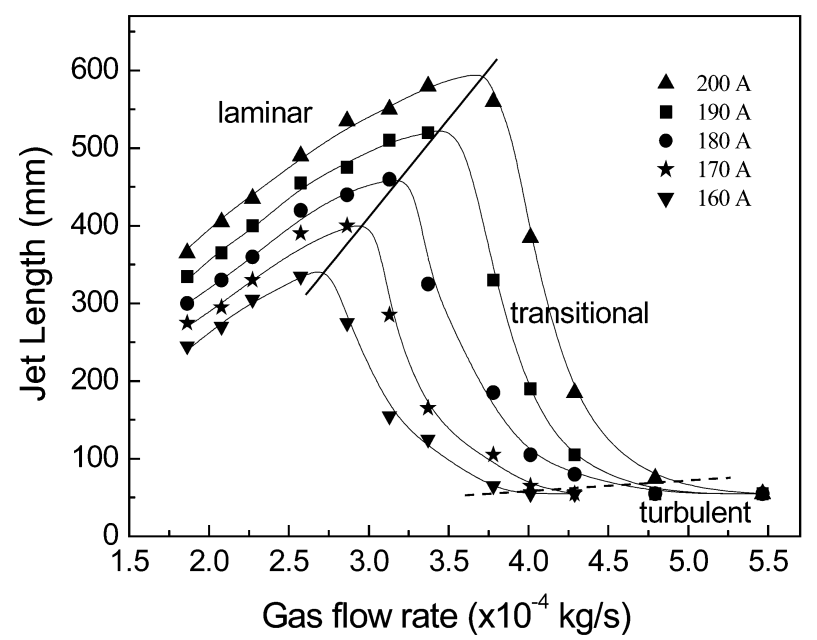

Fig. 2 Variations of the jet length with the argon flow rate for different arc currents.

rate. On the other hand, the jet length in the turbulent regime is much shorter and less dependent on the arc current or the argon flow rate. In the unsteady transitional regime, the time-averaged jet length decreases with increasing flow rate but appreciably increases with increasing arc current.

Figure 3 presents the variations with the arc current of the measured maximum plasma temperature and axial velocity at the laminar plasma jet axis and near the plasma torch exit. The maximum plasma temperature and axial velocity increase with increasing arc current. It is found that the highest plasma temperature and the maximum axial velocity near the torch exit can be as high as $17000 \mathrm{~K}$ and $1000 \mathrm{~m} / \mathrm{s}$, respectively. 


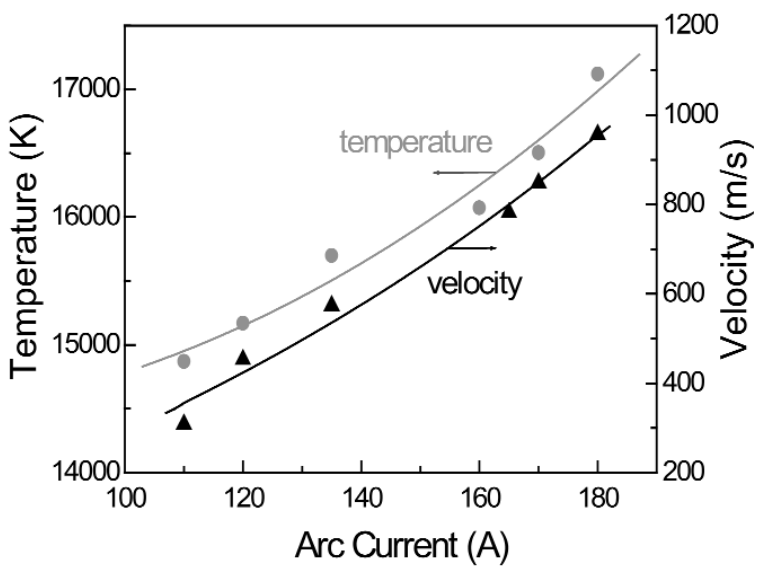

Fig. 3 Variations with the arc current of measured plasma temperature and axial velocity at laminar plasma jet axis and near the plasma torch exit. Argon flow rate: $1.6 \times 10^{-4} \mathrm{~kg} / \mathrm{s}$.

Experimental results show that there always exist fluctuations in the measured arc voltage and in the measured jet impact-pressure and spectral-emission intensity, even for the laminar flow case. Figure 4 compares typical experimental results concerning the variations with time of the measured impact-pressures in the laminar and turbulent plasma jets. The fluctuations with frequency of $300 \mathrm{~Hz}$ exist in both laminar and turbulent jets, but higher frequency and random fluctuations also appear in the turbulent plasma jet. The $300-\mathrm{Hz}$ fundamental waves in the fluctuations are caused by the alternative voltage components in the rectified DC power supply, as shown by the experimental results in Fig. 5, where the measured variations with time of the arc voltage are displayed. It is interesting to note that although there exist appreciable arc voltage and jet parameter fluctuations, the laminar plasma jet can still maintain its laminar flow state and its long high-temperature region appearance is steady. This fact implies that the laminar plasma jet can resist the interference caused by the fluctuations in arc voltage and in jet parameters.

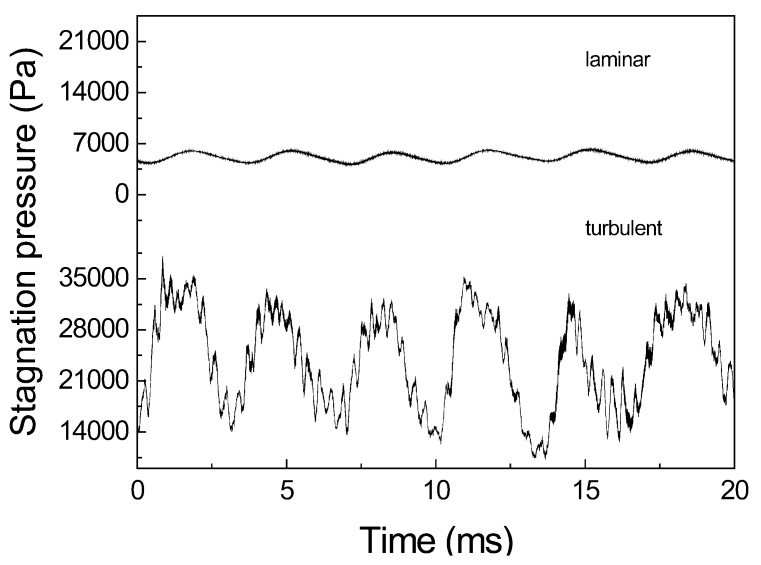

Fig. 4 Comparison of variations of impact pressure with time for laminar and turbulent plasma jets. Argon flow rate $2.4 \times 10^{-4} \mathrm{~kg} / \mathrm{s}$ for laminar jet, $3.8 \times 10^{-4} \mathrm{~kg} / \mathrm{s}$ for turbulent jet; arc current $180 \mathrm{~A}$. 


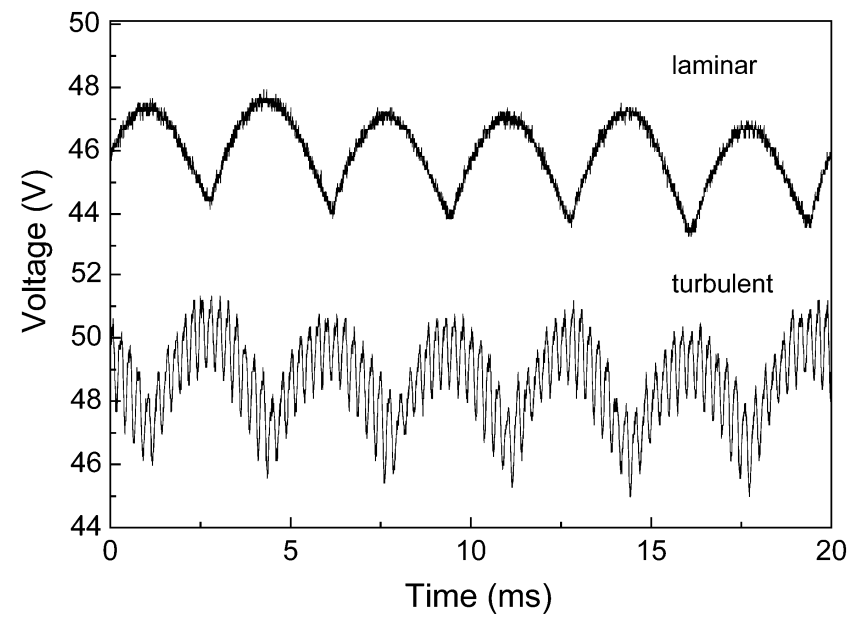

Fig. 5 Comparison of variations of arc voltage with time for laminar and turbulent plasma jets. Argon flow rate $2.4 \times 10^{-4} \mathrm{~kg} / \mathrm{s}$ for laminar, $4.0 \times 10^{-4} \mathrm{~kg} / \mathrm{s}$ for turbulent; arc current $180 \mathrm{~A}$.

It is found that the laminar plasma jet has good stiffness to withstand the impact of laterally injected particulate matter and its carrier-gas. Figures $6 \mathrm{a}$ and $6 \mathrm{~b}$ compare the long laminar plasma jet appearances observed for the cases without (a) and with (b) the lateral injection of alumina powder and its carrier gas. It is seen that due to the injection of the alumina powder and its carrier gas into the jet, the laminar plasma jet length somewhat shortens, but the laminar flow state of plasma jet can still be maintained and the deflection angle of the laminar plasma jet from the geometrical axis of plasma torch is very small. Similar results are also obtained in the modeling study [16], i.e., the laminar plasma jet appearance is less influenced by laterally injected carrier gas, as shown in Fig. 6c. 


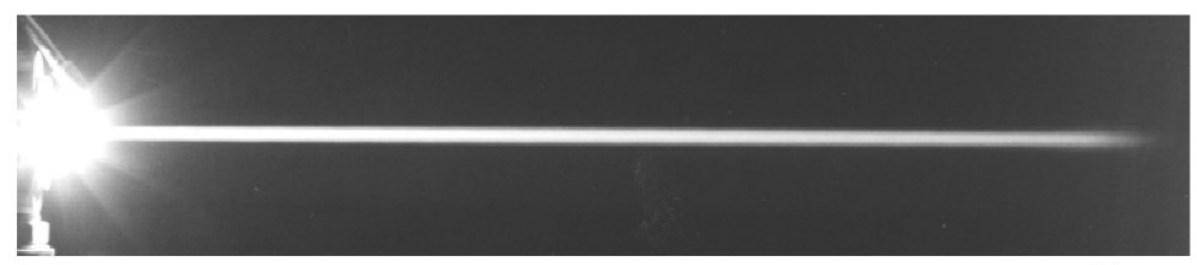

(a)

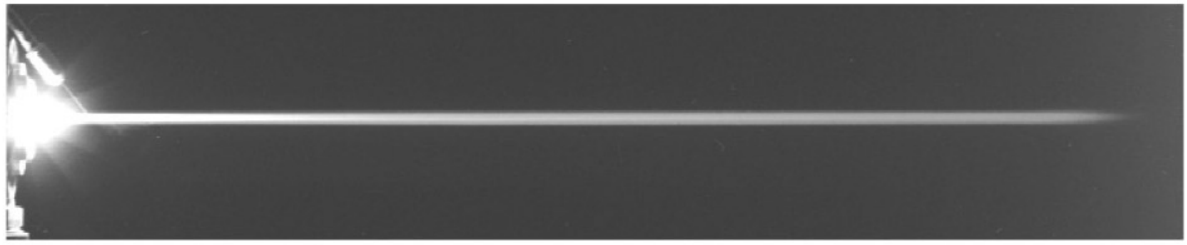

$50 \mathrm{~mm}$

(b)

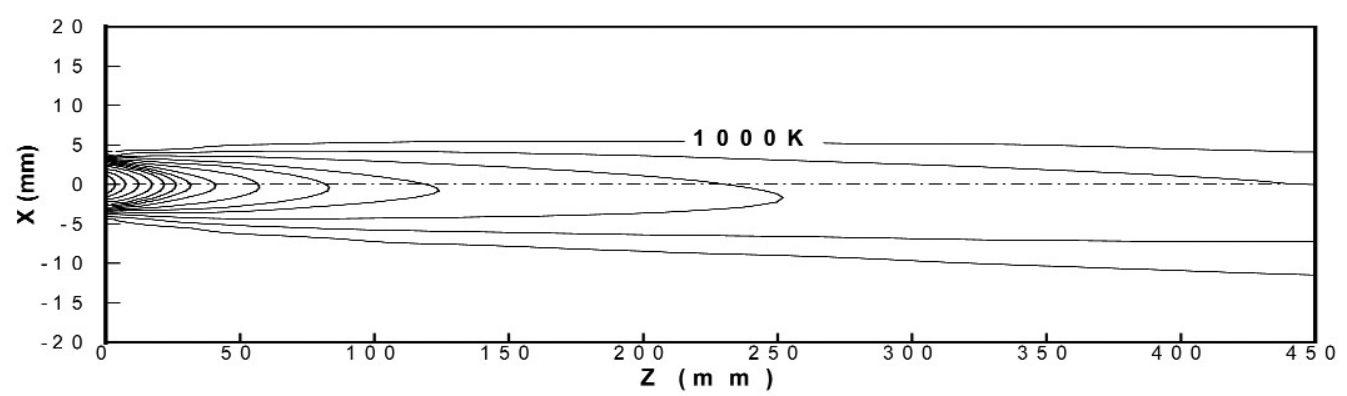

(c)

Fig. 6 Comparison of laminar plasma jet appearances for the cases without (a) and with (b,c) lateral carrier-gas injection. ( $\mathrm{a}$ and $\mathrm{b}$ ) experiment; (c) modeling.

\section{MODELING STUDIES}

The main assumptions used for the modeling study of the characteristics of argon plasma jets issuing into ambient air [16-20] include that the jet flow is steady, and the plasma is in the local thermodynamic equilibrium (LTE) state and optically thin to radiation. For the laminar plasma jets, the diffusion of argon in the argon-air mixture is handled by use of the combined-diffusion-coefficient method [21]. And mass, momentum, energy, and species (argon mass fraction) conservation equations with temperature- and composition-dependent plasma properties are solved numerically to predict the laminar plasma jet characteristics [16-19]. For predicting the turbulent jet characteristics, time-averaged physical quantities and combined molecular and turbulent transport coefficients are employed in the conservation equations. In addition, $K$ - $\varepsilon$ two-equation turbulence model is used for the turbulence modeling, and the turbulence-enhanced combined-diffusion-coefficient method is employed to deal with the entrainment of ambient air into the turbulent argon plasma jet [20].

The inlet radius $R$ of the plasma jet is taken to be $4 \mathrm{~mm}$, and the following radial $(r$-) distributions of plasma temperature and axial velocity are employed at the jet inlet:

$$
T=\left(T_{0}-T_{w}\right)\left[1-(r / R)^{2.3}\right]+T_{w}, \quad u=U_{0}\left[1-(r / R)^{1.4}\right]
$$

in which $T_{0}$ and $U_{0}$ are the highest temperature and the maximum axial velocity at the jet axis, respectively, whereas $T_{w}$ is the inner wall temperature of plasma torch $\left(T_{w}=700 \mathrm{~K}\right)$. The temperature and 
axial-velocity profiles (1) were employed in a few previous studies [20,22] and were shown to be able to predict plasma jet parameters (temperature, velocity, etc.) in reasonable agreement with correspondent experimental data for a typical turbulent argon plasma jet issuing into ambient air. For facilitating the comparison of laminar and turbulent plasma jet characteristics, the same inlet temperature and axialvelocity profiles are used for both the laminar and turbulent plasma jets in the present study. The computational domain, governing equations, boundary conditions, and other computation details, have been described elsewhere [16-20] and thus are not repeated here. Some typical modeling results are presented in the following to compare the characteristics of laminar and turbulent argon plasma jets issuing into ambient air.

The computed streamlines presented in Fig. 7 show that the entrained air flow rate and spreading angle for the laminar plasma jet are much smaller than their counterparts for the turbulent plasma jet. The computed isotherms presented in Fig. 8 show that the axial decreasing rate of plasma temperature in the laminar plasma jet is much less than that in the turbulent plasma jet, and thus the high-temperature region length of the laminar plasma jet is much longer than that of the turbulent plasma jet. For a fixed jet inlet temperature $T_{0}$, the laminar plasma jet length (e.g., expressed by the axial distance of $4000 \mathrm{~K}$ isotherm) increases notably with increasing jet inlet velocity, while the turbulent plasma jet length is much shorter and less influenced by the jet inlet velocity. The computed variations of argon mass fraction $\left(f_{A}\right)$ along the plasma jet axes presented in Fig. 9 show that the axial decreasing rate of $f_{A}$ in the laminar plasma jet is also much less than that in the turbulent plasma jet. As a result, $f_{A}$ on the laminar plasma jet axis can still be as great as $0.4-0.7$ ( $f_{A}$ increases with increasing jet inlet velocity) even at the axial distance as large as $400 \mathrm{~mm}$ from the jet inlet. On the other hand, $f_{A}$ has dropped to 0.3 at a much shorter axial distance, i.e., $40 \mathrm{~mm}$, for the turbulent plasma jets, and $f_{A}$ profiles along the turbulent plasma jet axis are less influenced by the jet inlet velocity. Similar computed results are also obtained for the cases with a fixed jet inlet velocity but different jet inlet temperatures. Namely, the axial decreasing rates of plasma parameters (temperature, axial velocity, and argon mass fraction) for the laminar plasma jet are appreciably less than those for the turbulent plasma jet. The $f_{A}$ and axial-velocity profiles along the turbulent plasma jet axes are less influenced by the jet inlet temperature.
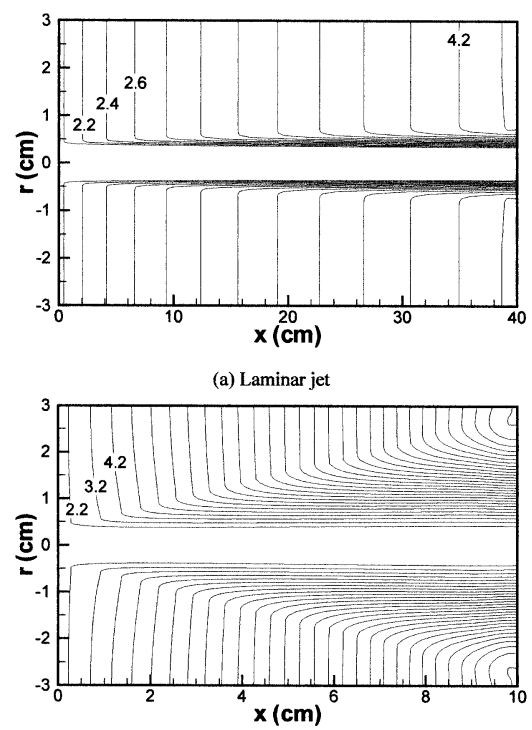

(b) Turbulent jet

Fig. 7 Computed streamlines of laminar and turbulent plasma jets. $U_{0}=1000 \mathrm{~m} / \mathrm{s}, T_{0}=13000 \mathrm{~K}$. Numerals in the figure express the values of stream function (in $10^{-4} \mathrm{~kg} /\left(\mathrm{s} \cdot\right.$ radian)); streamline interval: $0.2 \times 10^{-4} \mathrm{~kg} /(\mathrm{s} \cdot \mathrm{radian})$ for (a), and $1.0 \times 10^{-4} \mathrm{~kg} /(\mathrm{s} \cdot$ radian) for (b).

(C) 2006 IUPAC, Pure and Applied Chemistry 78, 1253-1264 


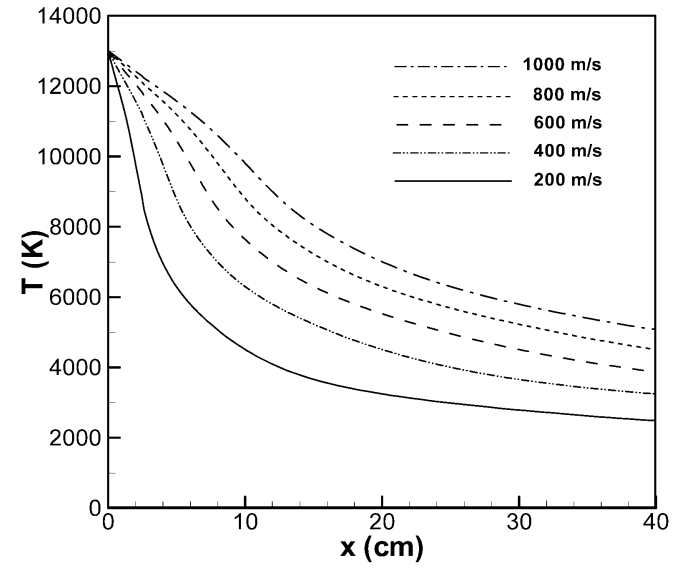

(a) Laminar jet

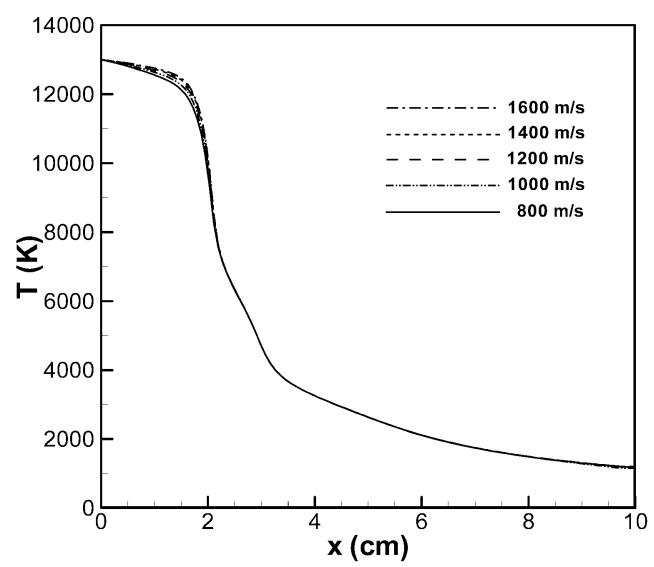

(b) Turbulent jet

Fig. 8 Comparison of the computed effects of jet inlet velocity on the plasma temperature distributions along the laminar (a) and turbulent (b) plasma jet axes $\left(T_{0}=13000 \mathrm{~K}\right)$.

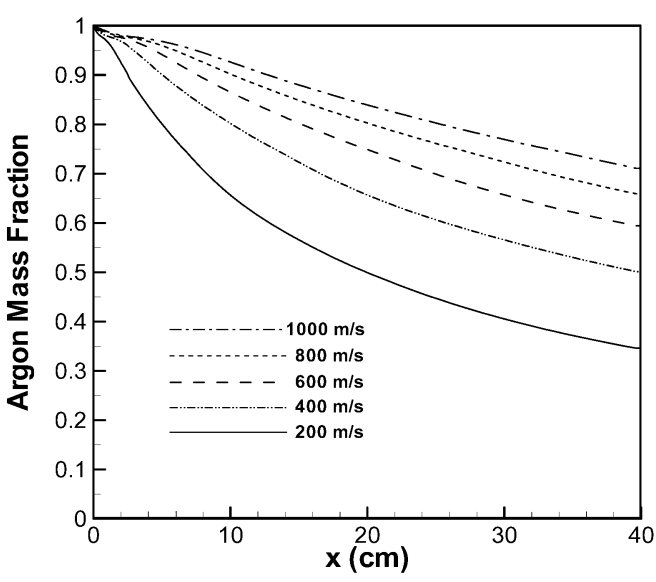

(a) Laminar jet

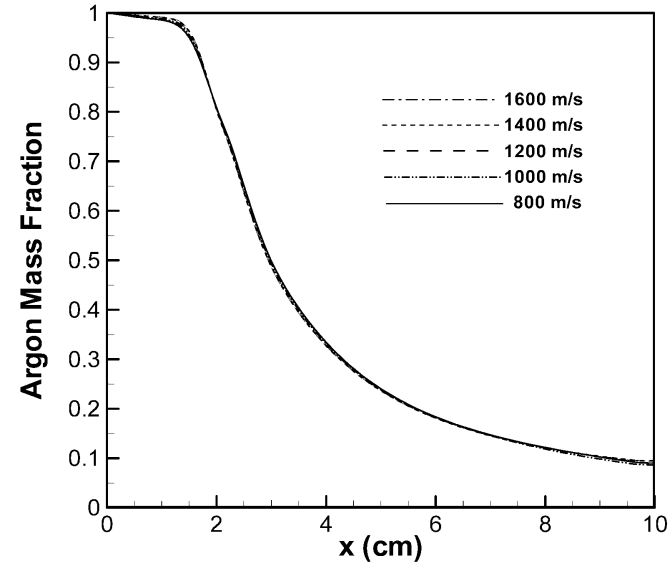

(b) Turbulent jet

Fig. 9 Comparison of the computed effects of jet inlet velocity on the argon mass fraction distributions along the laminar (a) and turbulent (b) plasma jet axes $\left(T_{0}=13000 \mathrm{~K}\right)$.

The modeling results are consistent with the experimental observation, including that the laminar plasma jet length is much longer and increases notably with the increase of the jet inlet velocity or temperature, while the turbulent plasma jet length is much shorter and less dependent on the jet inlet velocity or temperature.

The observed great difference between the laminar and turbulent plasma jet characteristics is due to their different air-entraining laws. That is, only molecular entraining mechanism exists in the laminar plasma jet, while turbulent transport is the dominant entraining mechanism for the turbulent plasma jet. For a cold laminar gas jet, analyses $[1,23]$ show that the entrained gas mass flow rate $G_{\text {entr }}=8 \pi \mu x$, i.e., $G_{\text {entr }}$ is directly proportional to the axial distance $x$ from the jet inlet and to the gas viscosity $\mu$, but independent of jet inlet velocity or jet inlet flow rate. On the other hand, for a cold turbulent gas jet, experimental results [24] show that the entrained gas mass flow rate $G_{\text {entr }}$ is directly proportional to the 
jet inlet velocity or jet inlet mass flow rate $G_{\text {in }}$, i.e., $G_{\text {entr }} / G_{\text {in }}=0.16(x / R)$, or the jet mass flow rate $G=$ $G_{\text {in }}+G_{\text {entr }}$ at the axial location $x$ can be expressed as $G / G_{\text {in }}=1+0.16(x / R)$. So far, no analytical or experimental results are available concerning the laws of surrounding gas entrainment into the laminar and turbulent plasma jets. The modeling study is thus conducted in this study. The computed results show that for the laminar plasma jet the entrained gas mass flow rate $G_{\text {entr }}$ increases with increasing viscosity of surrounding gas [18] and slightly depends on the jet inlet velocity or temperature (since gas viscosity depends on temperature for this case). On the other hand, $G_{\text {entr }}$ is approximately directly proportional to the jet inlet mass flow rate for the turbulent plasma jet. The computed variations with the axial distance $x$ of the jet mass flow rate normalized with respect to the jet inlet mass flow rate $\left(G / G_{\text {in }}\right)$ along the axes of laminar and turbulent plasma jets are compared in Fig. 10 for different jet inlet velocities. The results show that the values of $G / G_{\text {in }}$ for the turbulent plasma jet are much larger than that for the laminar plasma jet at the same downstream location. It means that the absolute value of the air mass flow rate entrained into the turbulent plasma jet $\left(G_{\text {entr }}\right)$ is much larger than that into the laminar plasma jet for a given axial distance $x$. The normalized entrained air mass flow rate (i.e., $G_{\text {entr }} / G_{\text {in }}$ or $\left.\left(G / G_{\text {in }}\right)-1\right)$ almost does not depend on the jet inlet mass flow rate $G_{\text {in }}$ (or jet inlet velocity $\left.U_{0}\right)$ for the turbulent plasma jet, implying that the absolute value of the entrained air flow rate $G_{\text {entr }}$ is approximately proportional to the jet inlet mass flow rate $G_{\text {in }}$ for the turbulent plasma jet. On the other hand, $G_{\text {entr }} / G_{\text {in }}$ notably decreases with increasing jet inlet velocity or jet inlet mass flow rate $G_{\text {in }}$ for the laminar plasma jet, implying that the absolute value of the entrained air flow rate is only slightly dependent on the jet inlet velocity. The computed variations of the normalized air mass flow rate entrained into laminar and turbulent plasma jets with the jet inlet temperature show that the normalized entrained air flow rate almost does not depend on the jet inlet temperature for the turbulent plasma jet, but somewhat increases with increasing jet inlet temperature for the laminar plasma jet (not shown here as separate figures).

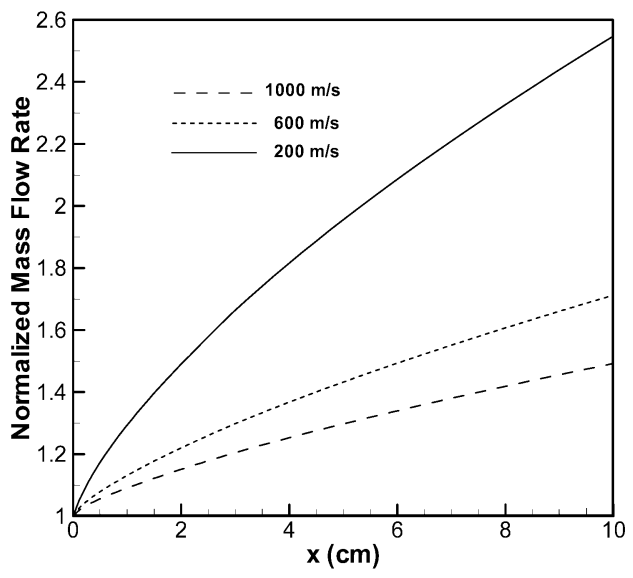

(a) Laminar

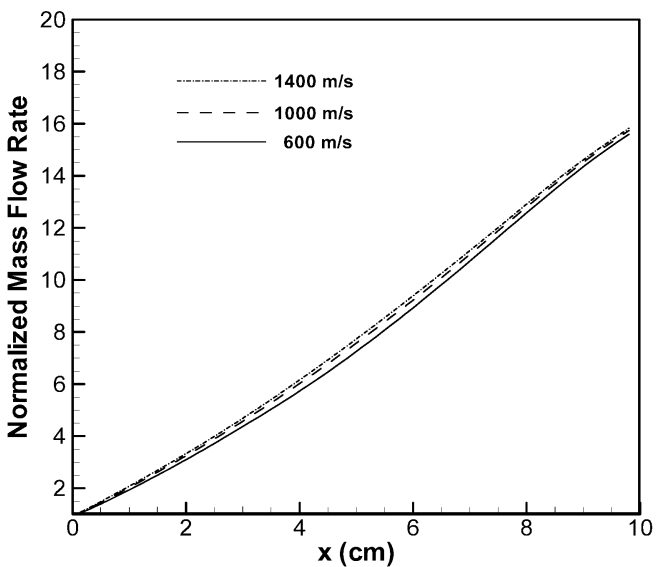

(b) Turbulent

Fig. 10 Variations with the axial distance of the normalized jet mass flow rates for laminar (a) and turbulent (b) argon plasma jets issuing into ambient air $\left(T_{0}=13000 \mathrm{~K}\right)$.

A temperature difference as great as $10^{4} \mathrm{~K}$ is always involved in the laminar plasma jet, and thus a question is often raised about whether natural convection may affect significantly the long laminar plasma jet characteristics? Modeling results concerning the effect of natural convection on the flow fields of laminar argon plasma jets downwards and upwards issuing into ambient air [17] show that the computed temperature, axial velocity, and argon mass fraction distributions within the typical laminar 
plasma jets are less influenced by the natural convection, only a little difference can be detected in the jet downstream region and near the jet outer edge in the computed streamlines. For the case that the laminar argon plasma jet is horizontally issuing into ambient air, the computed results demonstrate that the effect of natural convection on the laminar plasma jet appearance is also negligibly small [19]. Figure 11b presents the computed isotherms in the long laminar argon plasma jet issuing horizontally into ambient air. Noting that the scale of abscissa is much larger than that of ordinate, the upward bending of the plasma jet caused by natural convection is very small, in agreement with the experimental observation shown in Fig. 11a.
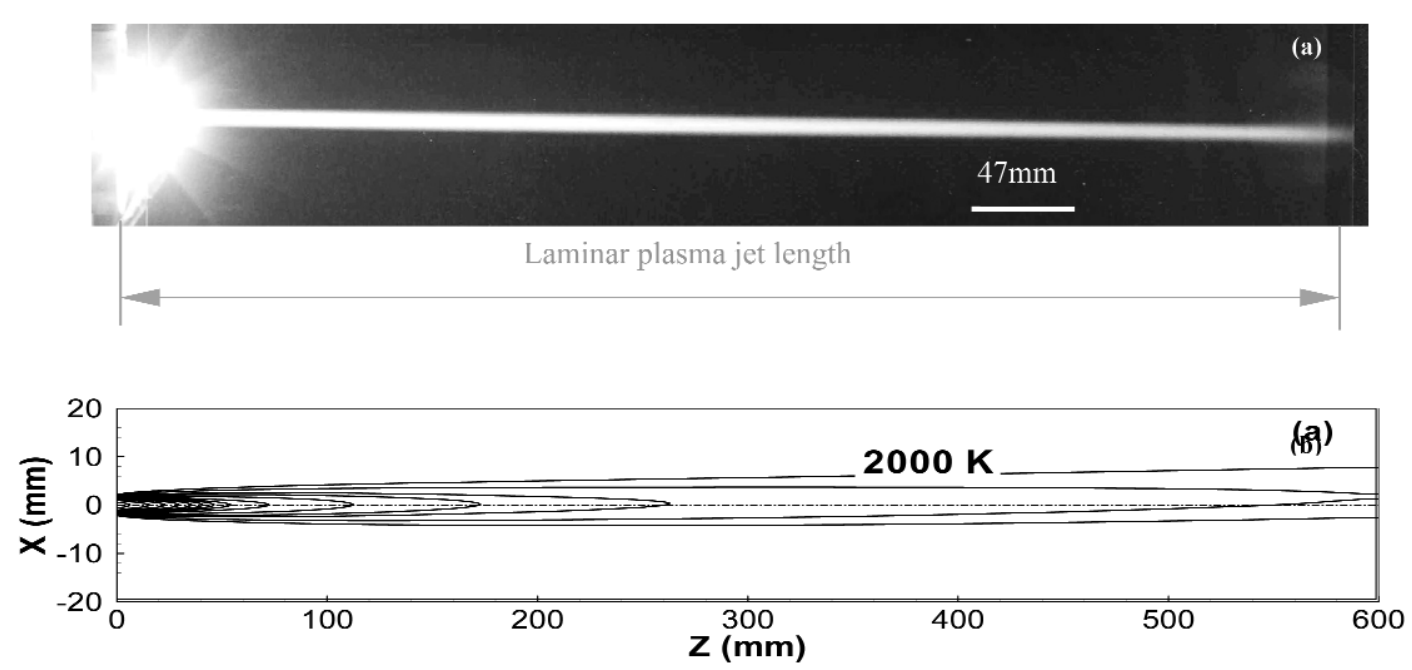

Fig. 11 Effects of natural convection on the characteristics of laminar argon plasma jet horizontally issuing into ambient air. (a) experimental observation; (b) modeling result.

Modeling results show that the long laminar plasma jet has good enough stiffness to endure the impact of the lateral injection of powders and their carrier gas. The laminar plasma jet can maintain its laminar flow state and assume only a small deflection (a few degrees) from its original axis due to the carrier-gas impact [16], as mentioned above associated with the discussion of Fig. 6. Particulate matter can thus be injected into the high-temperature region of laminar plasma jet with suitably adjusted injection parameters. Those predicted results also agree with experimental observation [13].

\section{PRELIMINARY APPLICATIONS}

Due to its attractive features, the long laminar plasma jet may become a useful tool for the thermal plasma science and technology. From the viewpoint of basic studies, the merit of the long laminar plasma jet is that there is no complexity caused by turbulence and its flow field parameters can be easily adjusted. However, so far not enough research efforts are devoted to apply the laminar plasma jet in this aspect. From the viewpoint of materials processing, extremely low noise environment, reduced ambient air entrainment, and adjustable jet flow field characteristics may improve the working surroundings of operators, enhance the process controllability and repeatability, and decrease the oxidation degree of processed metallic materials. Applying the laminar plasma jet to the preparation of thermal barrier coatings demonstrates that the prepared $\mathrm{ZrO}_{2}$ coatings assume fine microstructure, low surface roughness, and uniform pore distribution [11,14]. The remelting hardening of cast iron surface using laminar plasma jet shows good remelting process controllability, good surface morphologies, and stable and uniform hardening surface, while unstable and nonuniform hardening is often involved if the 
turbulent plasma jet remelting is employed $[10,12,13]$. Some additional experimental results are presented in Fig. 12 about the laminar plasma jet cladding of the stainless steel surface by adding Ni-alumina (a) and of the Al-Si alloy surface by adding SiC particles (b). It is found that a metallurgically bonded clad layer can be formed on the stainless-steel surface as seen in Fig. 12a, and the SiC particles can be well embedded into the Al-Si alloy surface layer as seen in Fig. 12b, as desired.
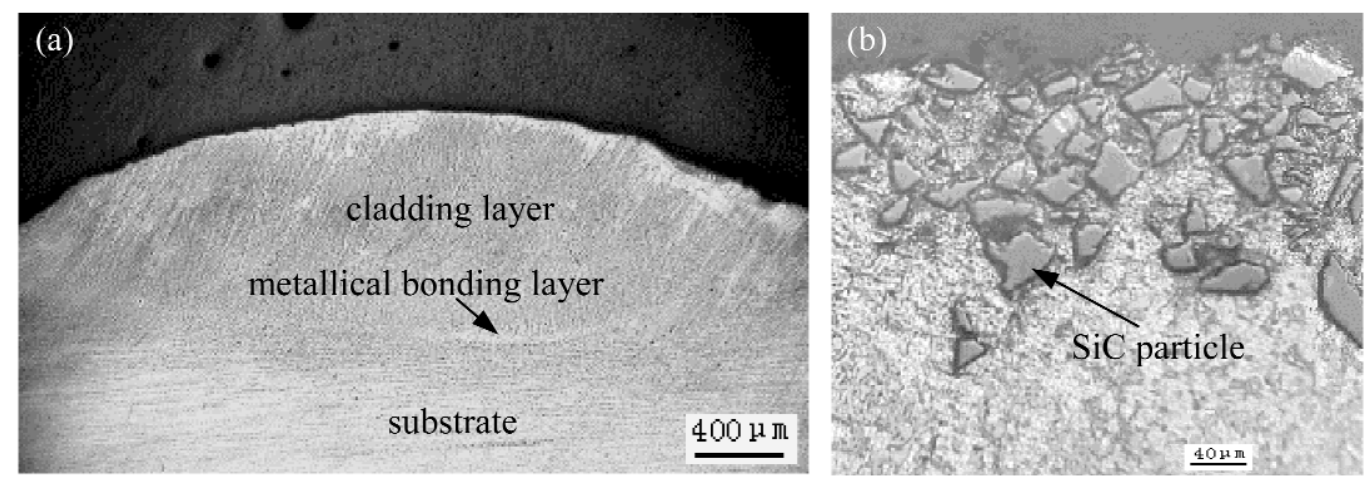

Fig. 12 Laminar plasma jet cladding of the stainless steel surfaces by adding Ni-alumina (a) and of the Al-Si alloy surface by adding $\mathrm{SiC}$ particles (b).

\section{UNSOLVED PROBLEMS}

Why can a long laminar plasma jet be generated and maintained? What is the physical mechanism? So far, these problems are still puzzling.

In fluid mechanics, usually Reynolds number $\operatorname{Re}=\rho u D / \mu$ is employed as the criterion to characterize the flow-state transition for a round gas jet, where $\rho, u, D$, and $\mu$ are the gas density, flow velocity, jet-nozzle diameter (or jet diameter) and viscosity, respectively. Thus, one might expect that there could be a constant value of the Reynolds number along the solid critical lines demarcating the laminar and transitional jet regimes in Figs. 1 and 2. Since the argon mass flow rate in the plasma torch can expressed as $G=(\pi / 4) D^{2} \rho u$, we can use $\operatorname{Re}=4 G /(\pi D \mu)$ to calculate the Reynolds number with the merit that $G$ and $D$ are unambiguously known and only $\mu$ is to be determined in a suitable way. We tried to use the integrally averaged value of $\mu$ between the temperatures at torch wall and at jet center to evaluate Re. Although reasonable Re values $\left(\sim 10^{3}\right)$ were obtained, the evaluated Re were not constant along the critical line. Then we turned to estimate $\mu$ at different characteristic temperatures (highest temperature, bulk temperature, the temperature corresponding to 10 or $20 \%$ boundary layer temperature drop, etc.), none of them could give satisfactory results. It seems that the Reynolds number is not the sole criterion to characterize the flow-state transition under thermal plasma conditions. However, determining what other criteria should be added to characterize the flow transition is still a problem. Tsygankov [25] reported the generation of a hard laminar flame with a laminar flame length/diameter ratio as large as 100 and the pertinent Reynolds number can be as high as $\operatorname{Re} \sim 10^{4}$. The reason for the hard laminar flame being generated was believed to be due to that a high-temperature burning flame-envelope was encircling the central flow of cold combustible mixture. The existence of a local high-temperature region near the jet-nozzle wall and in the jet/surrounding-gas mixing region would lead to the reduction of the Reynolds number evaluated at boundary temperature. However, for the laminar plasma jet, the gas temperature at the jet center is always much higher than that in the jet edge region, and thus the generation and maintenance mechanism of the laminar plasma jet is different from that of the hard laminar flame. It is anticipated that the flow and temperature fields within the DC arc plasma torch and near the jet inlet are important for the laminar plasma jet generation and maintenance. 


\section{ACKNOWLEDGMENT}

This work was supported by the National Natural Science Foundation of China (Nos. 50336010, 50276065, 10405015).

\section{REFERENCES}

1. H. Schlichting. Boundary Layer Theory, $7^{\text {th }}$ ed., pp. 230-234, McGraw-Hill, New York (1979).

2. K. S. Pasumarthi, A. K. Agrawal. Exp. Fluids 38, 541 (2005).

3. S. Jendoubi, P. J. Strykowski. Phys. Fluids 6, 3000 (1994).

4. P. Fauchais. J. Phys. D: Appl. Phys. 37, R86 (2004).

5. T. Honda, T. Takashi, A. Kanzawa. Int. J. Heat Mass Transfer 24, 1247 (1976).

6. X. Chen, X.-M. Chen. Chem. Eng. Commun. 87, 171 (1990).

7. V. I. Kuz'min, O. P. Solonenko, M. F. Zhukov. In Proc. $8^{\text {th }}$ National Thermal Spray Conf., 11-15 Sept. 1995, Houston, TX, pp. 83-88.

8. K. Osaki, O. Fukumasa, A. Kobayashi. Vacuum 59, 47 (2000).

9. W. X. Pan, W. H. Zhang, W. H. Zhang, C. K. Wu. Plasma Chem. Plasma Process. 21, 23 (2001).

10. W. X. Pan, W. H. Zhang, W. Ma, C. K. Wu. Plasma Chem. Plasma Process. 22, 271 (2002).

11. W. X. Pan, W. Ma, C. K. Wu. In Mechanics and Material Engineering for Science and Experiments, pp. 427-431, Science Press, Beijing (2001).

12. W. X. Pan, X. Meng, G. Li, Q. X. Fei, C. K. Wu. Surf. Coat. Technol. 197, 345 (2005).

13. W. X. Pan, G. Li, X. Meng, W. Ma, C. K. Wu. Pure Appl. Chem. 77, 373 (2005).

14. W. Ma, W. X. Pan, C. K. Wu. Surf. Coat. Technol. 191, 166 (2005).

15. E. Pfender. Plasma Chem. Plasma Process. 19, 1 (1999).

16. D.-Y. Xu, X. Chen, K. Cheng. J. Phys. D: Appl. Phys. 36, 1583 (2003).

17. K. Cheng, X. Chen. J. Phys. D: Appl. Phys. 37, 2385 (2004).

18. D.-Y. Xu, X. Chen. Int. Commun. Heat Mass Transfer 32, 939 (2005).

19. D.-Y. Xu, X. Chen. W. X. Pan. Int. J. Heat Mass Transfer 48, 3253 (2005).

20. K. Cheng, X. Chen. Int. J. Heat Mass Transfer 47, 5139 (2004).

21. A. B. Murphy. Phys. Rev. E 48, 3594 (1993).

22. (a) J. R. Fincke, D. M. Crawford, S. C. Snyder, W. D. Swank, D. C. Haggard, R. L. Williamson. Int. J. Heat Mass Transfer 46, 4201 (2003); (b) R. L. Williamson, J. R. Fincke, D. M. Crawford, S. C. Snyder, W. D. Swank, D. C. Haggard. Int. J. Heat Mass Transfer 46, 4215 (2003).

23. L. G. Loitsyanski. Laminar Boundary Layer, pp. 167-172, Physico-Mathematic Literature, Moscow (1963) (in Russian).

24. F. Ricou, D. B Spalding. J. Fluid Mech. 11, 21 (1961).

25. G. T. Tsygankov. Combust. Explosion Shock Wave 26, 695 (1991). 